\title{
EXPERIMENTAL EVALUATION OF THE UV RAMAN LIDAR SENSITIVITY IN DETECTION OF TRACES OF CHEMICAL COMPOUNDS
}

\author{
Sergey Bobrovnikov ${ }^{1,2 *}$, Evgeny Gorlov ${ }^{1,2}$, Viktor Zharkov ${ }^{1}$ \\ ${ }^{I}$ V.E. Zuev Institute of Atmospheric Optics, SB RAS, Tomsk, Russia, *Email: bsm@iao.ru \\ ${ }^{2}$ National Research Tomsk State University, Tomsk, Russia.
}

\begin{abstract}
Experimental results are presented on the remote detection of traces of some chemical compounds on the surface with the help of Raman lidar built on the basis of an excimer $\mathrm{KrF}$ laser with a narrow line emission and multi-channel spectrum analyzer based on the diffraction spectrograph and a time gated ICCD camera. The sensitivity of the system is evaluated for the sensing range of $10 \mathrm{~m}$. At the accumulation of the signal over 1000 laser pulses, the detection threshold of the nitrogencontaining chemical compounds of about of units of $\mu \mathrm{g} / \mathrm{cm}^{2}$ has been reached. The effect of the substrate material on the sensitivity of the Ramanlidar method for detecting traces of chemicals on the surface is analyzed.
\end{abstract}

\section{INTRODUCTION}

As previously shown in $[1,2]$, the lidar method based on the effect of Raman scattering in the ultraviolet (UV) wavelength range allows to achieve the sensitivity of about several ppm in detection of gaseous substances in the atmosphere. At the same time, the effect of Raman scattering allows to detect and identify substances not only in the gaseous phase, but also in the liquid and solid phases. Solution of the problem of development of the lidar method for the remote detection and identification of traces of chemical compounds on the surfaces of various items is of great practical and scientific interest and should be studied to assess its sensitivity, noise-stability, and the application field [3-5]. For this purpose, the work was done to upgrade and use the Raman-lidar method for the detection of traces of high-energy nitrogen-containing chemical compounds on the surface.

\section{UV RAMAN LIDAR}

The UV Raman lidar is built on the coaxial scheme. An excimer $\mathrm{KrF}$ laser with a narrow lasing line is used as the radiation source.

The scattered radiation reaches the receiving telescope aperture that creates an image of the scattering volume on the entrance slit of the spectrometer (Shamrock 500i). At the output of the spectrograph in the localization area of the spectrum, a cathode of a time gated ICCD camera is set that allows to detect the signal in the photon counting mode and in the mode of accumulation of charge. At the entrance of the spectrograph, an edge filter blocking the laser line of $248.3 \mathrm{~nm}$ is installed.

\section{EXPERIMENTAL SCHEME AND EVALUATION OF THE MAXIMUM LIDAR SENSITIVITY}

The Raman lidar for the detection of chemical compounds on the surface was tested in the laboratory room under standard conditions. The lidar transceiver system was focused at a distance of $10 \mathrm{~m}$, where a bracket with fixed samples possessing different surface concentrations of traces of chemical compounds was installed.

The width of the entrance slit of the spectrograph was $125 \mu \mathrm{m}$, which corresponds to the spectral resolution of $\sim 15 \mathrm{~cm}^{-1}$. The signal along the slit of the spectrograph was integrated. The signal was recorded in the photon counting mode at the accumulation over 1000 laser pulses.

At the initial stage of the experiment, the Ramanlidar spectrum response of the substrate was recorded. Figure 1 (curve 1) shows the experimental Raman spectrum recorded from the substrate (a clean microscope slide).

In all the recorded spectra, broad Raman bands of the glass components $\left(600 \mathrm{~cm}^{-1}\right.$ and 1150 $\mathrm{cm}^{-1}$ ) [6], as well as the vibrational-rotational Raman band of the atmospheric oxygen molecules $\left(1556 \mathrm{~cm}^{-1}\right)$ are observed. 
After the identification of the Raman spectra bands obtained in the experiments and subtraction of the substrate spectrum and Raman spectra of major atmospheric gases, the lidar response spectrum of traces of chemical substance deposited on the surface was selected.

The limit of the detectable concentration of traces was determined in the process of detecting the traces with a predetermined threshold surface concentration at a threshold value of the signal/noise ratio equal to 2 .
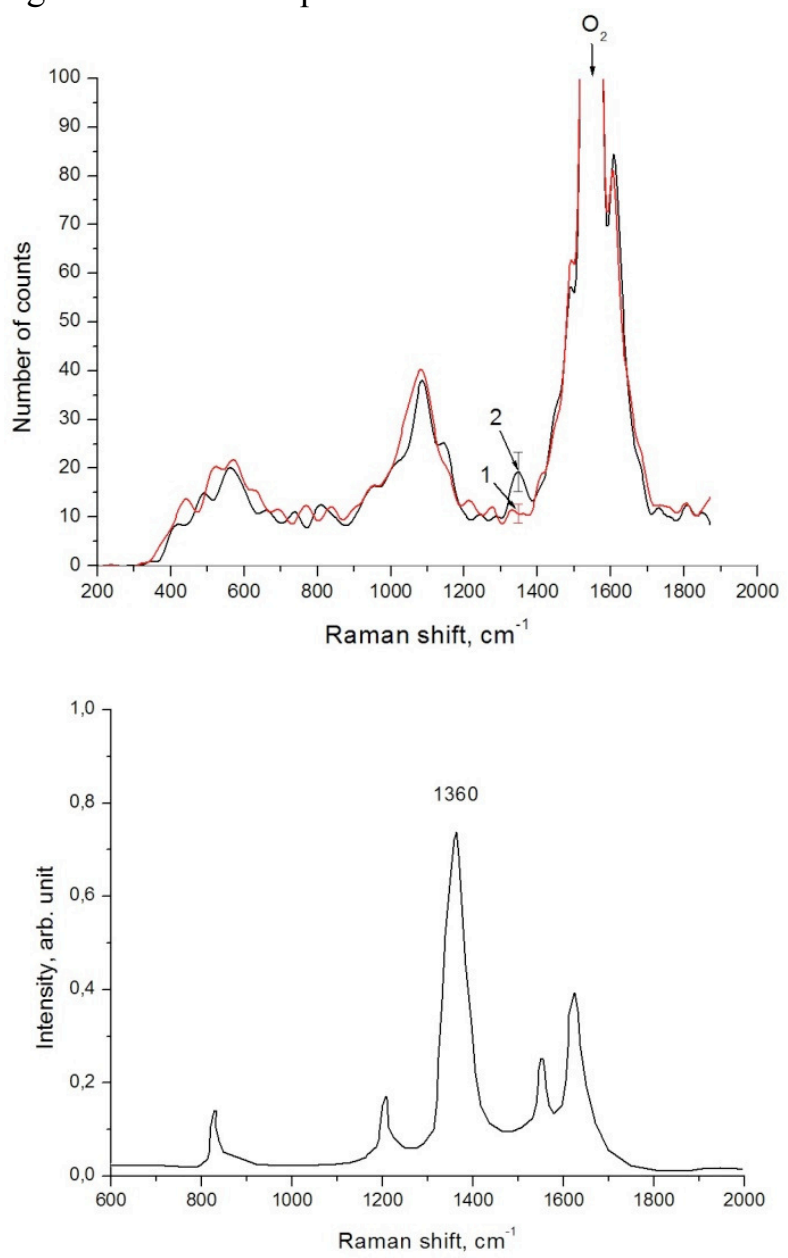

Fig. 1. Raman spectra of the substrate material (curve 1), of the TNT traces (curve 2) with the surface concentration of $500 \mathrm{ng} / \mathrm{cm}^{2}$ (upper figure) and the reference spectrum of TNT [7] (lower figure).

For the experiments, some high-energy nitrogen-containing substances with low volatility contained in the database of Raman spectra were used. When choosing the substances, the urgency of their remote detection was taken into account.
Figure 1 shows the results of experiments on the remote spectrometry of traces of trinitrotoluene (TNT) by the Raman method: curve 1 - Raman spectrum of the glass slide material; curve 2 Raman spectrum of the TNT traces deposited on a glass slide.

The signal observed in the region of the Raman shifts of $1500-1700 \mathrm{~cm}^{-1}$ is due to the vibrational-rotational Raman band of the atmospheric oxygen molecules.

It follows from the experiments on determining the sensitivity of the Raman lidar using the data on the detection of traces on the silica glass substrates (the glass microscope slides) that the limits of the detectable concentrations are $0.5 \mu \mathrm{g} / \mathrm{cm}^{2}$ for TNT, $25 \mu \mathrm{g} / \mathrm{cm}^{2}$ for pentaerythritol tetranitrate, and $20 \mu \mathrm{g} / \mathrm{cm}^{2}$ for ammonium nitrate.

It is obvious that the possible superposition of Raman bands of the substrate material on the Raman bands of the detected substances can significantly limit the sensitivity of the method. That is why, it is very important to know the spectroscopic properties of expected substrates for a more accurate assessment of the real limit of the detectable concentration and determination of the Raman-method application field.

\section{INFLUENCE OF THE SUBSTRATE ON THE SENSITIVITY OF THE RAMAN- LIDAR METHOD FOR DETECTING TRACES}

To investigate the noise level of the substrates, a series of experiments on the assessment of the luminescence intensity of the substrate surfaces, when exposed to KrF-laser radiation, was performed.

An analysis of experimental data showed that the noise level in the recorded region of the spectrum depends on the optical properties of the substrate material. For example, substrates with negligible absorption of the exciting radiation $(248 \mathrm{~nm})$ give the Raman bands, whose intensities are 3-5 orders of magnitude greater than those of the Raman signals of the traces (depending on the ratio between the substrate thickness and that of the substance layer under study).

Obviously, this effect can be attributed to a large depth of the radiation penetration into the substrate material, and consequently, to a large 
total volume of interaction. Figure 2 shows the Raman spectrum of an optical quartz glass KU-1.

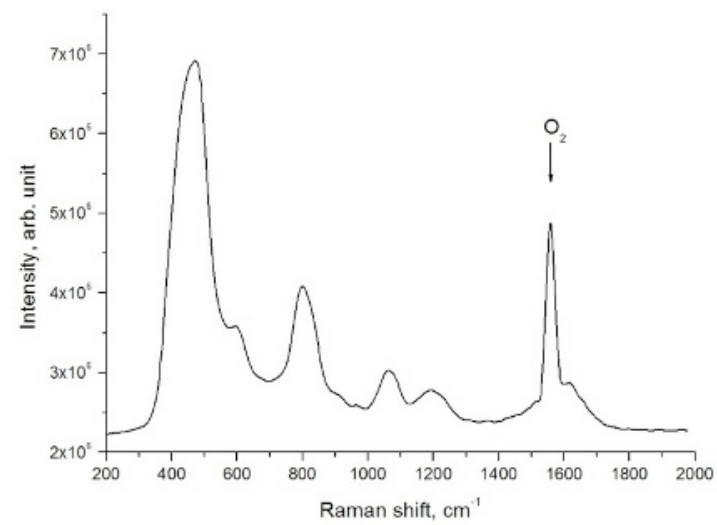

Fig. 2. Raman spectrum of the quartz glass KU-1.

In the weakly absorbing substrates, fluorescence is observed, whose spectrum covers the area of the frequency shifts and extends beyond this area. In this case, a substantial resonant rise of the Raman scattering intensity in the substrate material can be observed (Figure 3).
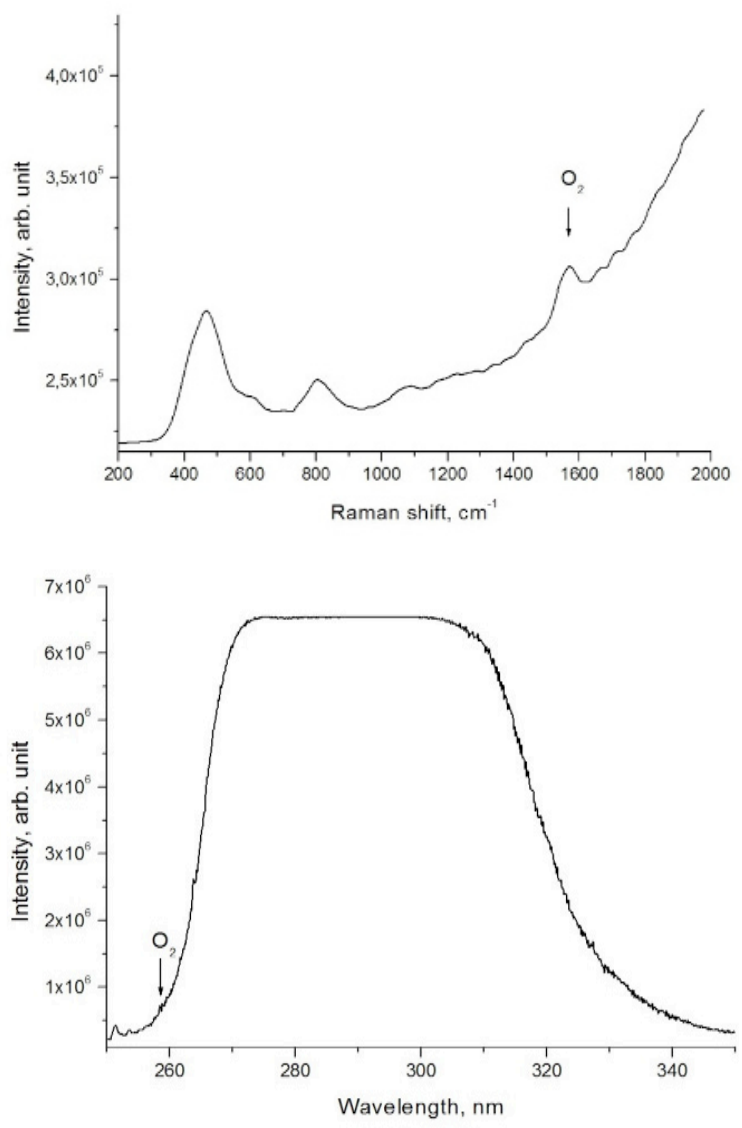

Fig. 3. Raman (upper figure) and fluorescence (lower figure) spectra in the quartz glass $\mathrm{KV}$.
In contrast, in the highly absorbing substrates, a decrease in the intensity of Raman bands of the substrate material is observed due to the decrease in the penetration depth. As shown by experiments, in this case, the intensity of Raman signals in the substrate material is comparable with the intensity of Raman signals in the traces of the tested substances (Fig. 1).

In the strongly absorbing substrates, an intense fluorescence was observed due to the high level of absorption of the exciting radiation in the substrate material (Fig. 4). However, the maximum of the fluorescence band intensity is shifted to the red region of the spectrum. The spectral region of the Raman shifts is free of noise.
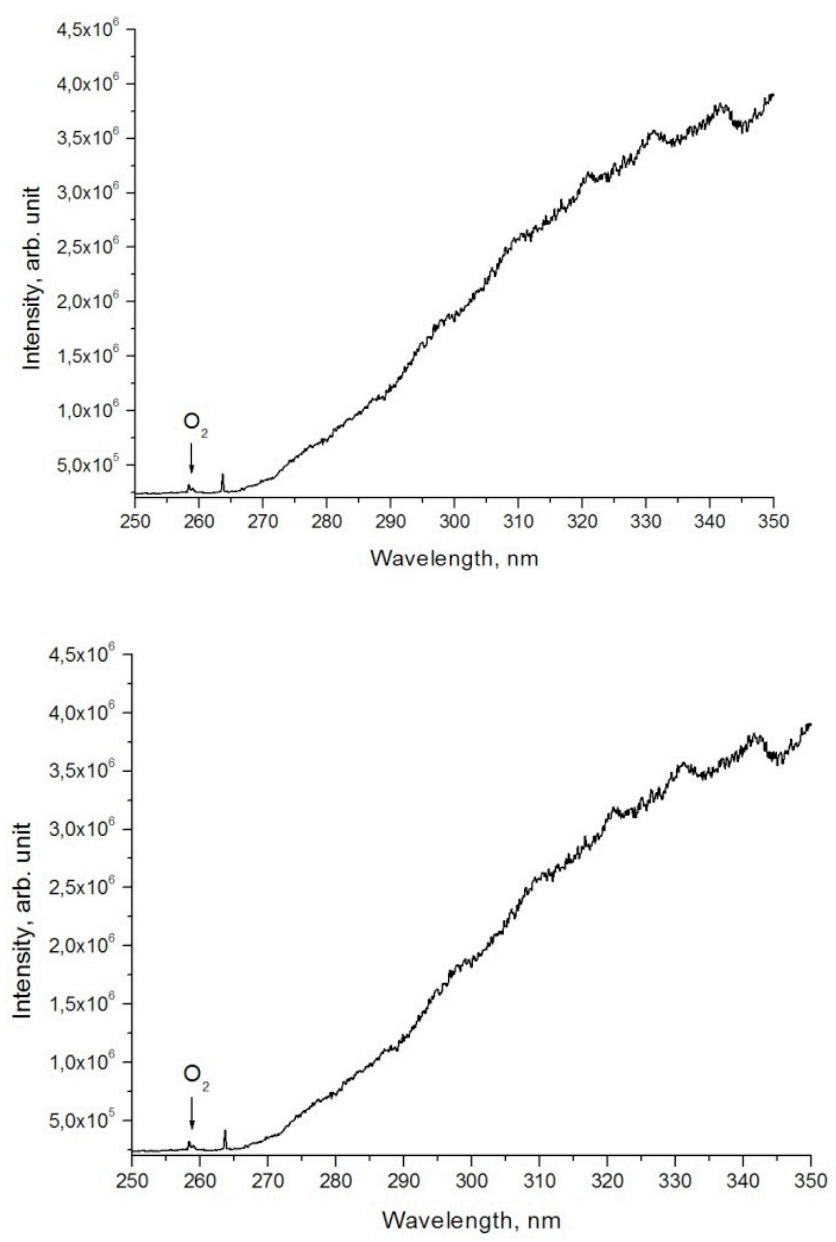

Fig. 4. Fluorescence spectra of the blue cotton (upper figure) and white cotton (lower figure). 


\section{CONCLUSIONS}

As a result of the statistical analysis of the data, it was found that the UV Raman lidar based on an excimer $\mathrm{KrF}$ laser provides reliable detection of traces of chemical compounds with the surface density of about several $\mu \mathrm{g} / \mathrm{cm}^{2}$, depending on the Raman cross section of the detected molecules.

It is found that the sensitivity of the method depends on the optical properties of the substrate material. Substrates transparent to the exciting radiation, as a rule, limit the sensitivity of the method due to the high efficiency of the Raman processes and broadband fluorescence.

\section{ACKNOWLEDGEMENT}

This work was supported in part by the RFBR under Grant No. 13-05-00096a and No. 13-0898013, the RF President grant No. NSH4714.2014.5 and No. MK-6286.2015.10, and the Russian Science Foundation (Agreement No. 1427-00022).

\section{REFERENCES}

[1] Bobrovnikov, S. M., E. V. Gorlov, V. I. Zharkov, 2012: Deep UV Raman lidar gas analyzer for environment monitoring, Proc. of the 26th ILRC 2012, Porto Heli, Greece, pp. 215-217.

[2] Bobrovnikov, S. M., E. V. Gorlov, V. I. Zharkov, 2013: Experimental estimation of the sensitivity of the UV Raman lidar, Atmos. Ocean. Opt., 26 (01), pp. 70-74.

[3] Carter, J. C., S. M. Angel, M. LawrenceSnyder, J. Scaffidi, R. E. Whipple, and J. G. Reynolds, 2005: Standoff Detection of High Explosive Materials at 50 Meters in Ambient Light Conditions Using a Small Raman Instrument, Appl. Spectrosc., 59(6), pp. 769-775.

[4] Jander, P. and R. Noll, 2009: Automated Detection of Fingerprint Traces of High Explosives Using Ultraviolet Raman Spectroscopy. Appl. Spectrosc. 63(5), pp. 559563.

[5] Moros, J., J. A. Lorenzo, K. Novotný and J. J. Laserna, 2013: Fundamentals of stand-off Raman scattering spectroscopy for explosive fingerprinting, J. Raman Spectrosc., 44, pp. 121130.
[6] Seuthe, T., M. Grehn, A. Mermillod-Blondin, H. J. Eichler, J. Bonse, and M. Eberstein, 2013: Structural modifications of binary lithium silicate glasses upon femtosecond laser pulse irradiation probed by micro-Raman spectroscopy, Opt. mater. express, 3(6), pp. 755-764.

[7] Gaft, M., L. Nagli, 2008: UV gated Raman spectroscopy for standoff detection of explosives, Opt. Mater., 30, pp. 1739-1746. 